\title{
Lasertherapie bei Prostatahyperplasie
}

— Im Vergleich zu herkömmlichen Operationen ist die Laserbehandlung einer benignen Prostatahyperplasie (BPH) eine wenig traumatisierende, schmerzarme und komfortable Alternative. Beim modernen Ceralas $^{\oplus}$ HPD Dual-Laser wird selektive Lichtvaporisation verwendet, um überflüssiges Weichgewebe schonend abzutragen (Ablationsrate von $3 \mathrm{~g}$ pro Minute). Aufgrund der hohen Absorption des Lasersystems in Wasser wird vermieden, dass Laserstrahlen versehentlich in die Blase und/ oder andere nicht zu behandelnde Bereiche emittiert werden. Der Ceralas ${ }^{\circledast}$ HPD Dual ist der erste urologische Laser der Welt mit zwei parallelen Wellenlängen von $1470 \mathrm{~nm}$ und $980 \mathrm{~nm}$. In Kombination mit der neuen, patentierten TWISTER ${ }^{\text {TM}}$-Faser gewährleistet das biolitec-Lasersystem nur minimale bis gar keine intra- oder postoperativen Blutungen sowie Schonung des umliegenden Gewebes zugunsten einer schnellen Rekonvaleszenz. Die Kontaktfaser TWISTER $^{\text {TM }}$ erlaubt eine Laser-TUR auf effiziente Weise. Das patentierte Design der Glasspitze gibt dem Anwender direk- tes, taktiles Feedback der abzutragenden und zu vaporisierenden Oberfläche und vereinfacht das Modellieren des Gewebes. Sogar größere Prostatadrüsen können in kurzer Zeit behandelt werden. Der Laser erzielt hohe Ablationsraten von $3 \mathrm{~g} / \mathrm{min}$ und und führt zu einer geringen aber effektiven Koagulationszone, wodurch mögliche Nebenwirkungen minimiert werden. Die Behandlung kann ambulant durchgeführt werden.

Im Franziskus Krankenhaus in Berlin wird der Ceralas ${ }^{\circledR}$ HPD Dual-Laser zur BPH-Therapie eingesetzt. Das Franziskus Krankenhaus bietet ferner auch Schulungen für den sicheren Gebrauch mit dem innovativen Ceralas $^{\oplus}$ HPD- Laser für die Behandlung der benignen Prostatahyperplasie an. Diese Trainings wurden bereits von Ärzten aus Japan, England, Frankreich, den USA, Russland und weiteren Ländern besucht.

Nach Informationen von biolitec, Jena

\section{Erektile Dysfunktion: neues Sildenafil-Generikum}

- Zentiva, die europaweite Generika-Marke von Sanofi, bietet mit Sildenafil Zentiva $^{\oplus}$ ab sofort eine Behandlung für Männer mit erektiler Dysfunktion. Der selektive PDE-5-Inhibitor ist bioäquivalent und indikationsgleich zu seinem Original Viagra ${ }^{\circledR}$. Es handelt sich bei dem Generikum um blaue, teilbare Filmtabletten. Der Wirkstoff hemmt den Abbau von cGMP in den glatten Gefäßmuskelzellen. So führt Sildenafil bei sexueller Stimulation zu erhöhten Blutspiegeln von cGMP im Corpus cavernosum und damit zu einer verstärkten Erektion. Ohne eine sexuelle Stimulation und Aktivierung des NO/cGMP-Systems löst der Wirkstoff keine Erektion aus. Sildenafil Zentiva ${ }^{\circledast}$ ist in allen Wirkstärken wie das Original Viagra ${ }^{\circledR}$ erhältlich: Zur Auswahl stehen 25 mg, 50 mg und 100 mg Filmtabletten in den Packungsgrößen 4 und 12 Filmtabletten.

Somit steht Männern mit Erektionsstörungen nun eine effektive und kostengünstige Therapie zur Verfügung. Die Verpackung ermöglicht aufgrund ihrer Gestaltung außerdem eine diskrete Abgabe. Weitere Informationen finden Interessierte unter www.potenzratgeber.info.

Nach Informationen von

Sanofi-Aventis, Frankfurt/Main 